\title{
ESTUDIO DEL DESGASTE EROSIVO POR CAVITACIÓN DE UN ACERO AUSTENÍTICO DE ALTO NITRÓGENO APOYADO EN EL USO DE LA DIFRACCIÓN DE ELECTRONES RETROPROYECTADOS-EBSD
}

\author{
ASSESSMENT OF CAVITATION-EROSION WEAR OF A HIGH NITROGEN \\ AUSTENITIC STAINLESS STEEL FROM ELECTRON BACKSCATTERING \\ DIFFRACTION-EBSD
}

\author{
Dairo Hernán Mesa Grajales ${ }^{1}$ \\ Carlos Mario Garzón Ospina² \\ Recibido 23 de marzo de 2009, aceptado 16 de agosto de 2010 \\ Received: March 23, 2009 Accepted: August 16, 2010
}

André Paulo Tschiptschin ${ }^{3}$

\begin{abstract}
RESUMEN
Muestras de acero inoxidable con estructura austenítica y contenido aproximado de nitrógeno de $0,9 \%$ en peso en solución sólida fueron sometidas a ensayos de cavitación vibratoria en agua destilada, con el fin de estudiar la evolución del desgaste erosivo por cavitación $(E C)$ a escala del tamaño de grano (mesoescala). Las muestras fueron obtenidas por medio de nitruración gaseosa a alta temperatura $(H T G N)$ del acero inoxidable dúplex $U N S S 31803$, las cuales fueron caracterizadas por difracción de electrones retroproyectados $(E B S D)$, a fin de obtener información de la orientación cristalina de los granos en la superficie a ser cavitada. La técnica de microscopía electrónica de barrido (SEM) fue usada para acompañar el proceso de desgaste para diferentes tiempos de ensayo. Como resultado de los ensayos de cavitación se determinó la pérdida de masa y se hizo un estudio secuencial de la apariencia superficial de las muestras para determinar los mecanismos de desgaste operantes en cada etapa. Los resultados fueron analizados a la luz de la caracterización cristalográfica previa. Para propósitos de comparación, fue usado el acero austenítico convencional UNS S30403. A partir de los resultados se observó que tanto la nucleación del daño como su evolución se dan de forma heterogénea a escala del tamaño de grano, resultado atribuido a la anisotropía en la deformación plástica. El carácter heterogéneo del daño por cavitación fue atribuido principalmente a las características de los límites de grano y a la microtextura dentro de los granos.
\end{abstract}

Palabras clave: Aceros inoxidables de alto nitrógeno, desgaste erosivo por cavitación, difracción electrónica por retrodispersión, EBSD, mesotextura.

\section{ABSTRACT}

Stainless steel samples with $100 \%$ austenitic microstructure and alloyed with $0.9 \mathrm{wt}$-\% of nitrogen in solid solution were tested under vibratory cavitation experiments in distilled water to study the cavitation-erosion wear (EC) at grain size level (mesoscale). Fully austenitic samples were obtained by high temperature gas nitriding (HTGN) a commercially available dúplex stainless steel, UNS S31803. The samples were characterized by electron backscattering diffraction, EBSD to obtain the crystalline orientation of individual grains. In addition, mass loss measurements were obtained and surface damage evolution was analyzed by scanning electron microscopy (SEM) at different times of the test. These results were analyzed as a function of the prior crystallographic characterization. For comparison, a conventional austenitic stainless steel, UNS S30403, was also investigated. It was observed that both the nucleation and the growth of damage are heterogeneous at the grain-size scale due to mesoscale plasticity anisotropy, induced during the CE tests. The heterogeneous character of the cavitation damage is related to both the grain boundary character and the microtexture inside the grains.

Keywords: High nitrogen stainless steels, cavitation erosion wear, electron backscattering diffraction-EBSD, mesotexture.

\section{INTRODUCCIÓN}

La difracción de electrones retroproyectados (EBSD) es una técnica que a pesar de ser relativamente moderna (desarrollada en la década de 1990) se ha convertido en pilar para el estudio, a escala micro y nanométrica, de aspectos relacionados con la cristalografía de materiales de ingeniería, tanto en el campo científico como industrial.

\footnotetext{
Profesor Asociado Universidad Tecnológica de Pereira. Colombia. E-mail: dhmesa@usp.br. Tel +55 1130915237 , Fax:+55 1130915237.

2 Profesor Auxiliar, Universidad Nacional de Colombia, Sede Bogotá. Colombia. E-mail: cmgarzono@unal.edu.co. Tel + 57 1 3165000 Ext. 3165000.

3 Profesor Titular Escola Politécnica da Universidade de São Paulo. Brasil. E-mail: antschip@ usp.br. Tel. +55 11 30915656 , Fax:+55 11 30915656.
} 
Entre los estudios que pueden ser abordados por esta técnica están: textura (orientación cristalina preferencial), tamaño de grano, tipo y cantidad de fases (incluyendo partículas de precipitados), desorientación entre dos o más granos, análisis de esfuerzos en regiones deformadas, como por ejemplo derivados de una laminación o a través de ensayos de desgaste por cavitación, etc., con resolución espacial de entre $0,05 \mu \mathrm{m}$ y $0,5 \mu \mathrm{m}[1-2]$.

Adaptado a microscopios electrónicos del tipo barrido (scanning electron microscopy: SEM) o transmisión (transmission electron microscopy: MET), el sistema EBSD combina estudios realizados con esos microscopios, como análisis metalográfico utilizando electrones secundarios (secondary electrons: SE) o electrones retrodispersos (back-scattered electrons: BSE) y microanálisis químico por espectrometría de energía dispersiva (energy dispersive spectrometer: EDS) y espectrometría por dispersión de longitud de onda (wavelength dispersive X-ray spectroscopy: WDS) para complementar los resultados obtenidos. Los datos EBSD son procesados y manipulados con ayuda de una técnica denominada microscopía de orientación de imagen (orientation image microscopy: OIM) [1, 3-8], a partir de la cual se obtienen mapas de distribución de orientaciones cristalográficas, llamados en este trabajo mapas OIM. El principio de funcionamiento y los elementos principales que conforman un sistema EBSD son presentados en la Figura 1.

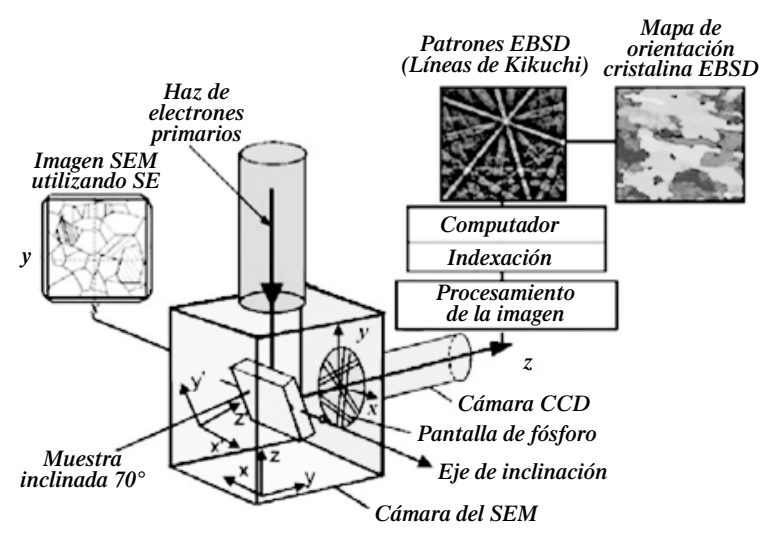

Figura 1. Principio de funcionamiento, elementos principales y posicionamiento de la muestra en un sistema EBSD acoplado a un SEM [2].

Con respecto a desgaste por cavitación, este es un fenómeno que afecta principalmente partes y equipamientos encargados de manipular o transportar fluidos, donde las piezas son damnificadas por un fenómeno conocido como erosión por cavitación (CE) [9].
Durante el tiempo que se ha estudiado la cavitación, han sido varios los materiales y tratamientos térmicos, termomecánicos y termoquímicos utilizados en la búsqueda para mejorar la resistencia a este tipo de desgaste. Por el lado de los materiales también han sido ensayadas las más variadas familias, como fundiciones de hierro, aleaciones a base de níquel y cobalto, aceros inoxidables, aleaciones, no ferrosas, etc. [10-12]. En el caso de tratamientos térmicos y termoquímicos, la nitruración gaseosa en alta temperatura, recubrimientos vía plasma y spray térmico, temple y revenidos sucesivos, etc., se cuentan entre los procedimientos más utilizados para aumentar la resistencia a la EC de materiales de ingeniería.

Para el caso específico de los aceros inoxidables de alto nitrógeno, ha sido reportado por investigadores [1316] que su elevado nivel de resistencia a la EC se debe principalmente al efecto que el nitrógeno tiene sobre la disminución de la energía de defecto de apilamiento, a la redistribución y aumento en la cantidad de dislocaciones, a las transformaciones de fase inducidas por la EC y al aumento de la plasticidad y del endurecimiento por deformación en estos materiales.

El objetivo de este trabajo es analizar la evolución del desgaste por cavitación, a nivel del tamaño de grano, en un acero inoxidable austenítico de alto contenido de nitrógeno con ayuda de las técnicas EBSD y SEM.

\section{DESARROLLO DE LA EXPERIMENTACIÓN}

La composición química de los aceros usados en este estudio es presentada en la Tabla 1.

Tabla 1. Composición química de los aceros UNS S31803 y UNS S30403.

\begin{tabular}{|c|c|c|c|c|c|c|c|c|}
\hline Material & $\mathrm{C}$ & $\mathrm{Cr}$ & $\mathrm{Ni}$ & $\mathrm{Mn}$ & $\mathrm{N}$ & $\mathrm{Cu}$ & $\mathrm{Si}$ & $\mathrm{Mo}$ \\
\hline UNS S31803 & 0,019 & 23,0 & 5,4 & 1,9 & 0,16 & 0,14 & 0,50 & 2,5 \\
\hline UNS S30403 & 0,04 & 18,7 & 9,6 & 0,95 & --- & -- & 0,03 & -- \\
\hline
\end{tabular}

\section{Nitruración gaseosa en alta temperatura}

Este tratamiento, conocido como high temperature gas nitriding: HTGN, fue realizado con el propósito de transformar un acero inoxidable dúplex (fases: austenita y ferrita), en un acero monofásico de fase $100 \%$ austenítica, el cual es menos susceptible al endurecimiento por deformación.

Se usaron muestras cuadradas de aproximadamente $2 \mathrm{~cm}^{2} \mathrm{de}$ área y $1,5 \mathrm{~mm}$ de espesor. Las condiciones del tratamiento fueron: temperatura de $1.200{ }^{\circ} \mathrm{C}$, tiempo de 8 horas y una presión de nitrógeno gaseoso de alta pureza de 1 atmósfera. Con estos parámetros se garantizó que el nitrógeno quedara dentro del material en la forma de solución sólida y no 
de nitretos, obteniéndose aproximadamente un contenido homogéneo de $0,9 \% \mathrm{~N}_{2}$ en peso (medido por WDS), tal como se predice del diagrama de fases para este acero y que no se muestra en este trabajo.

Con la nitruración gaseosa se obtuvo entonces un material de fase $100 \%$ austenítica, con tamaño de grano promedio de aproximadamente $160 \mu \mathrm{m}$ de diámetro y una estructura con alto grado de textura superficial, donde la mayoría de los granos (cristales) contiene la familia de planos (101) paralela a la superficie.

\section{Preparación de muestras y aplicación de la técnica EBSD}

Las muestras fueron preparadas metalográficamente, primero con papeles abrasivos (lijas), con tamaño de grano desde 220 hasta 2.500 , seguido por pulimento fino en paños con solución de diamante hasta tamaño de $1 \mu \mathrm{m}$ y finalmente con sílice coloidal con tamaño medio de $0,4 \mu \mathrm{m}$, durante aproximadamente 2 horas, utilizando una pulidora metalográfica automática.

Seguidamente fue realizado un barrido en un área previamente identificada. Para ello se usó un equipamiento EBSD de la TexSEM, acoplado a un SEM Philips XL30. El barrido se realizó en un área de 1,2 × 1,2 mm, con un paso de $2 \mu \mathrm{m}$ de distancia entre cada punto analizado. Con ayuda del software que acompaña la técnica EBSD (TSL®) se obtuvo un mapa de los granos dentro del área barrida, en el que es posible identificar, a través de un código de colores (IPF: inverse pole figure), la orientación cristalina de cada uno de esos granos.

\section{Ensayos de desgaste}

Para la realización de los ensayos de desgaste se usó el método de cavitación indirecta (muestra posicionada debajo de la punta del equipamiento de desgaste por cavitación), en un equipo telesonic ultrasonic modelo SG22 1.000/2.000G. Las muestras fueron ensayadas a cavitación por un periodo total de 64 horas. A diferentes intervalos de tiempo el ensayo era interrumpido para hacer el seguimiento de la pérdida de masa usando una balanza de precisión de $0,01 \mathrm{mg}$ y con esto determinar el desgaste del material. A cada interrupción del ensayo la muestra era llevada al SEM, donde se identificaba el área barrida por EBSD para ser fotografiada y acompañar la evolución del daño causado por la cavitación desde su fase inicial hasta el tiempo máximo de ensayo (64 h). La Figura 2 (a) muestra el esquema de un equipamiento de cavitación, donde se indican sus partes principales, las cuales son descritas en la Tabla 2. En la Figura 2 (b) se presenta una fotografía en detalle donde se indica el posicionamiento de la muestra.

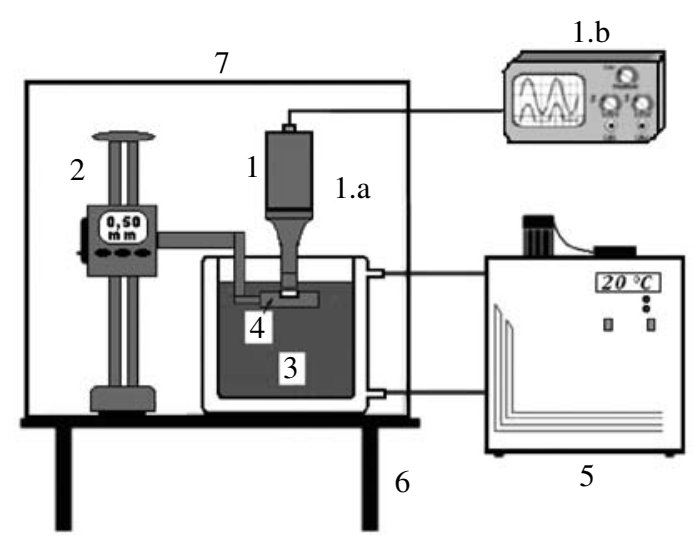

(a)

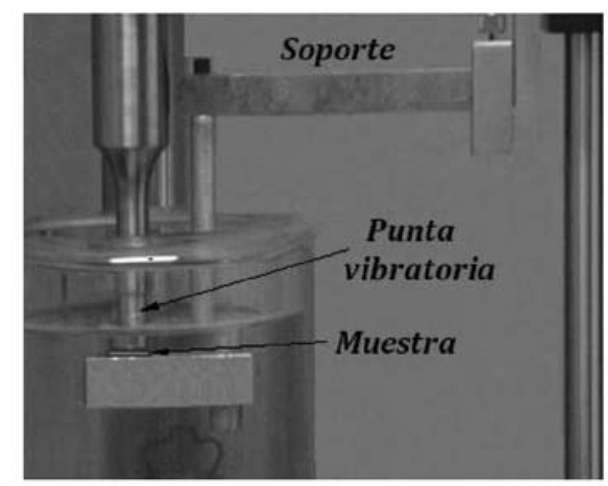

(b)

Figura 2. Equipamiento (cavitómetro) usado en los ensayos de cavitación ultrasónica (a) esquema general, (b) detalle del portamuestras.

Tabla 2. Descripción de las partes que componen un cavitómetro. La numeración de la columna 1 es correspondiente con la numeración de la Figura 2 (a).

\begin{tabular}{|c|c|c|}
\hline Item & \multicolumn{2}{|l|}{ Descripción } \\
\hline 1 & $\begin{array}{l}\text { Módulo vibratorio, } \\
\text { Telsonic SG } 1000\end{array}$ & $\begin{array}{l}\text { 1.a. Transductor y actuador } \\
\text { 1.b. Generador y controlador. }\end{array}$ \\
\hline 2 & \multicolumn{2}{|c|}{$\begin{array}{l}\text { Soporte magnético con brazo articulado con encaje y } \\
\text { reloj comparador. }\end{array}$} \\
\hline 3 & \multicolumn{2}{|c|}{$\begin{array}{l}\text { Recipiente con pared falsa (camisa) para refrigeración, } \\
\text { fabricación artesanal. }\end{array}$} \\
\hline 4 & \multicolumn{2}{|c|}{ Portamuestras, fabricación artesanal. } \\
\hline 5 & \multicolumn{2}{|c|}{ Baño termostático, Quimis modelo Q-214M2 } \\
\hline 6 & \multicolumn{2}{|c|}{ Soporte, fabricación artesanal. } \\
\hline 7 & \multicolumn{2}{|c|}{ Cámaras de aislamiento acústico, construcción artesanal. } \\
\hline
\end{tabular}

\section{RESULTADOS Y DISCUSIÓN}

\section{Nitruración gaseosa y técnica EBSD}

Las Figuras 3 (a) a la (c) muestran una secuencia de imágenes del acero UNS S31803 donde se observa: (a) la micrografía de este acero después de la nitruración, 
(b), el mapa de distribución de orientaciones cristalinas OIM obtenido en el área barrida y (c) una micrografía SEM, después que la muestra fue ensayada a EC por 4 horas, del área correspondiente al mapa EBSD de la Figura 3 (b).

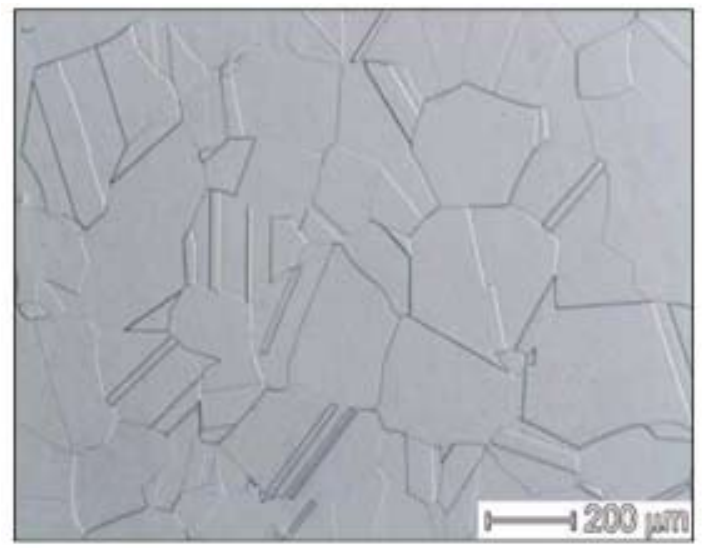

(a)

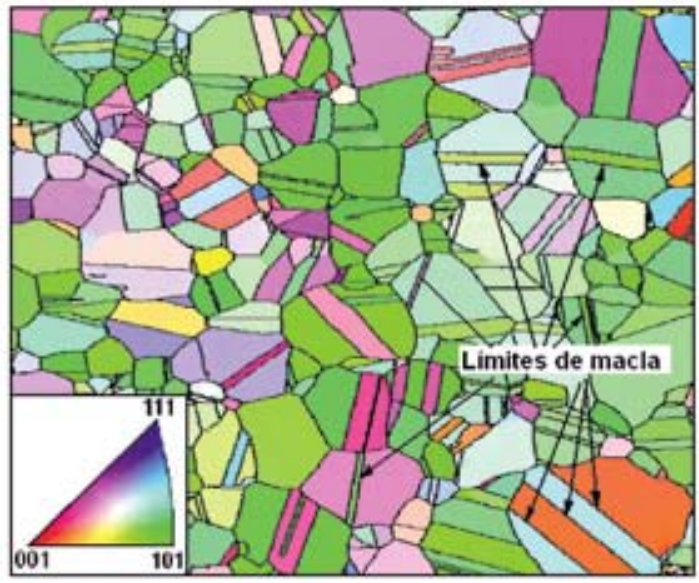

(b)

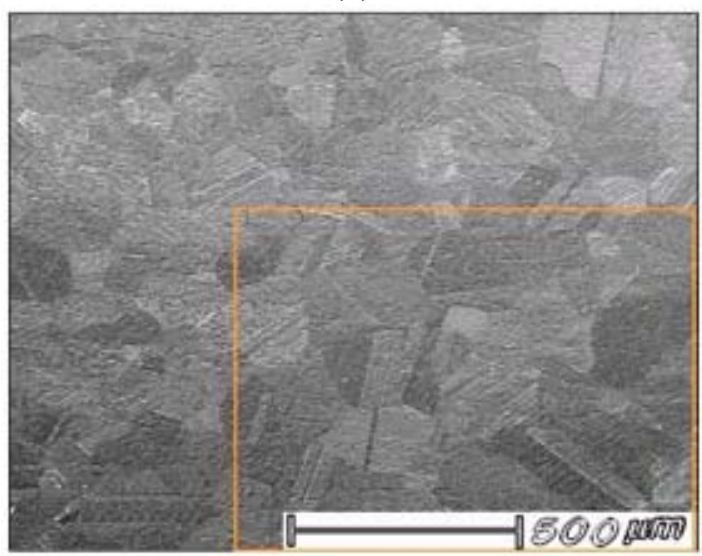

(c)

Figura 3. (a). Estructura austenítica obtenida después de la nitruración, (b) mapa EBSD y (c) micrografía SEM cavitada 4 horas, correspondiente al área EBSD en (b).
La Figura 3 (a) muestra una microestructura austenítica típica, en este caso obtenida por nitruración gaseosa en alta temperatura del acero UNS S31803. La obtención de esa microestructura, diferente a la microestructura dúplex del material de partida, se debe al fuerte efecto estabilizador de la austenita que ejerce el nitrógeno cuando se encuentra en solución sólida.

En la Figura 3 (b) se muestra un mapa de distribución de orientaciones cristalinas OIM (Orientation Imaging map) de este acero, donde se observa que la gran mayoría de los granos presenta color verde que, según la nomenclatura de la figura de polo inversa (triángulo de colores colocado en la parte inferior izquierda de la Figura 3 (b)), corresponden a las orientaciones cristalográficas de granos cuyos planos atómicos corresponden con la familia (101) orientados paralelos a la superficie del material.

En la Figura 3 (c) se presenta una micrografía SEM de la superficie cavitada por 4 horas, región correspondiente al área mapeada por EBSD y mostrada en la Figura 3 (b). En esta micrografía se evidencia que el desgaste sufrido por los granos del material se presenta de forma heterogénea. Hecho que puede ser confirmado por la presencia de granos que se muestran más conservados que otros (regiones más oscuras). De la micrografía de la Figura 3 (c) también se nota que el desgaste ocurre de forma heterogénea a nivel de los límites de grano, afectando en mayor proporción los límites de macla (límites rectos dentro de los granos), más que otros tipos de límites de grano.

\section{Evolución del desgaste por cavitación}

Las Figuras 4 (b) a (f) muestran la evolución del proceso de desgaste por cavitación sufrido por el material durante las 64 horas de ensayo a las que fueron sometidas las diferentes muestras. Cada micrografía corresponde al tiempo de ensayo en que este fue interrumpido para determinar la pérdida de masa, es decir, para 4, 9, 25, 49 y 64 horas. Las micrografías para 16 y 36 horas no se muestran, aunque esos tiempos también fueron utilizados para realizar la medición de la pérdida de masa. La Figura 4 (a) presenta el mapa de distribución de orientaciones cristalinas OIM obtenido de la superficie de la muestra antes de ser sometida a ensayos de desgaste EC. En el mapa OIM se puede observar la orientación cristalina de cada grano donde se confirma que después de la nitruración a alta temperatura se obtuvo un material con un alto grado de textura hacia la familia de planos (101) paralelos a las superficie. Esta área se corresponde con la parte de la micrografía mostrada en la Figura 3 (c) encerrada por recuadro. Las Figuras 5 (a) a la (d) muestran una secuencia de imágenes, similares a las presentadas en la Figura 4, sólo que para el acero UNS S30403, usado como material de comparación, estas figuras son: un mapa OIM y tres micrografías SEM para $0,5,2$ y 3 horas de ensayo EC. 

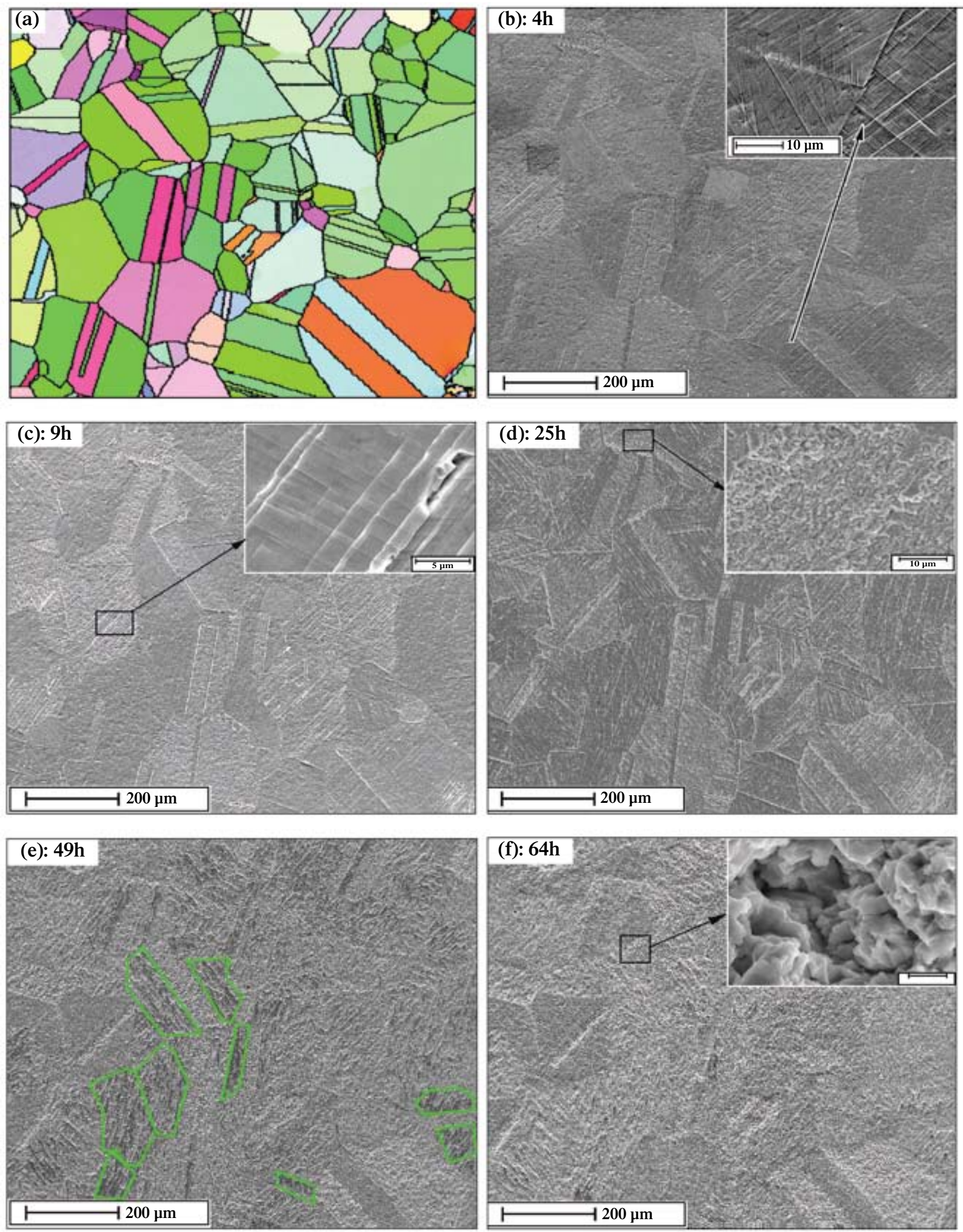

Figura 4. Evolución del proceso de desgaste por cavitación en el acero UNS S31803. (a) mapa EBSD correspondiente al área analizada y (b) a la (f) micrografías SEM para 4, 9, 25, 49 y 64 horas de ensayo respectivamente. 
En las micrografías de las Figuras 4 (b) y (c), para tiempos de ensayo de 4 y 9 horas respectivamente, se observa que los primeros síntomas de desgaste en la superficie del material se presentan a nivel de límites de grano, concentrándose especialmente en los límites de macla. El daño es acompañado por deformación plástica dentro de los granos, la cual puede ser evidenciada en el cambio de rugosidad de la superficie y en la presencia de bandas de deslizamiento (slip lines) dentro de algunos de ellos. Estas bandas de deslizamiento pueden ser observadas en la micrografía ampliada en el recuadro superior derecho de la Figura 4 (b).

Para tiempos de ensayo entre 9 y 25 horas, las líneas de deslizamiento, localizadas al interior de los granos, comienzan a destacarse de manera importante en el proceso de desgaste como muestran las Figuras 4 (c) y (d). En el detalle anexo a la Figura 4 (c) se evidencia también la presencia de fractura frágil como mecanismo principal de remoción de masa en regiones altamente deformadas; sin embargo, para ese tiempo de ensayo la pérdida de masa aún no es suficientemente alta para ser detectada por la balanza utilizada (ver Figura 6 para el acero UNS S31803). Por otro lado, regiones que todavía no fueron afectadas por la fractura continúan deteriorándose por deformación plástica, donde la formación de picaduras y microgrietas llevan a la superficie a un estado tal de deformación que los esfuerzos repetidos provenientes de la implosión de burbujas de vapor y microchorros formados durante el proceso de cavitación provocan fácilmente la remoción de material. El cambio de mecanismo de desgaste, de deformación plástica para desgaste por fractura, provoca pérdida de masa a través del desprendimiento de partículas de desgaste de la superficie, conocidas como debris. Esa pérdida de material es causada por la acción de los campos de esfuerzos de tipo cortante provenientes de la implosión de burbujas de cavitación.

Para tiempos intermediarios de ensayo, arriba de $25 \mathrm{~h}$, existe una competencia entre mecanismos de desgaste, donde la deformación plástica deja de ser el mecanismo de desgaste principal para dar paso a la fractura frágil, después que la superficie ha alcanzado un elevado estado de fatiga, con la subsiguiente pérdida de masa en la forma de debris. En este estado, el desgaste del material ocurre predominantemente por arranque de microplaquetas, como puede ser observado en el detalle colocado en la Figura 4 (d). En este detalle también se hace evidente la existencia del mecanismo de fatiga, en donde se observan marcas típicas de este fenómeno en las paredes de los granos cuyos vecinos ya fueron arrancados.
Para las etapas finales del desgaste (tiempos de ensayo mayores a 49 horas), donde se alcanza una tasa constante de pérdida de masa en el acero UNS S31803, el endurecimiento por deformación, típica de aceros inoxidables austeníticos y la fatiga sufrida por la superficie seguida por fractura frágil, hace que el material sea deteriorado rápidamente. Estos eventos son similares en el acero UNS S30403, aunque en este último se presenta de manera más rápida. Para estas últimas etapas de desgaste el mecanismo principal de daño es la fractura frágil derivada de fatiga de bajos ciclos. Evidencias de trazos de fatiga y fractura frágil pueden ser observadas en el detalle colocado en la Figura 4 (f).

Una característica importante que puede ser observada en la secuencia de micrografías de la Figura 4 (d) y (e) y en los detalles anexos, es la forma heterogénea en que el desgaste actúa, tanto en los límites de grano como en su interior, pues es claro que no todos los límites ni todos los granos se desgastan equitativamente. Esto demuestra que las tensiones provenientes de la implosión de burbujas de cavitación actúan de forma independiente. Este fenómeno puede ser verificado en las Figuras 4 (d) y (e), donde se observan granos completamente dañados y granos con su superficie bastante conservada (regiones oscuras) aún para altos tiempos de ensayo.

De igual forma, se observa que granos bastante deteriorados están ubicados al lado de otros prácticamente intactos, probablemente debido a la diferencia de orientación cristalina entre ellos, la cual actúa como atenuadora o aceleradora de las tensiones provenientes de la cavitación, siendo que los límites de macla, al presentar una más rápida acumulación de tensiones, restringen la deformación, permitiendo la actuación del mecanismo de fatiga y fractura, siendo los primeros a deteriorarse $[10,16]$.

La alta resistencia al desgaste de granos con orientación (101) paralela a la superficie, como los mostrados en la Figura 4 (e), como hipótesis, puede ser atribuida a la forma como los esfuerzos provenientes de las ondas de choque y microchorros, generados después de la implosión de las burbujas de cavitación, alcanzan los granos. Donde para esa orientación específica (101) es altamente probable que el esfuerzo proyectado (esfuerzo de cizallamiento crítico), aquel capaz de desplazar planos atómicos y llevar al material a deformarse plásticamente, no es necesario que sea alcanzado, pues el estado de deformación de la superficie es tan alto que valores más bajos al esfuerzo de cizallamiento crítico son suficientes para provocar mucho daño a granos con otras orientaciones cristalinas, los que además se encuentran en alto grado de fatiga. 

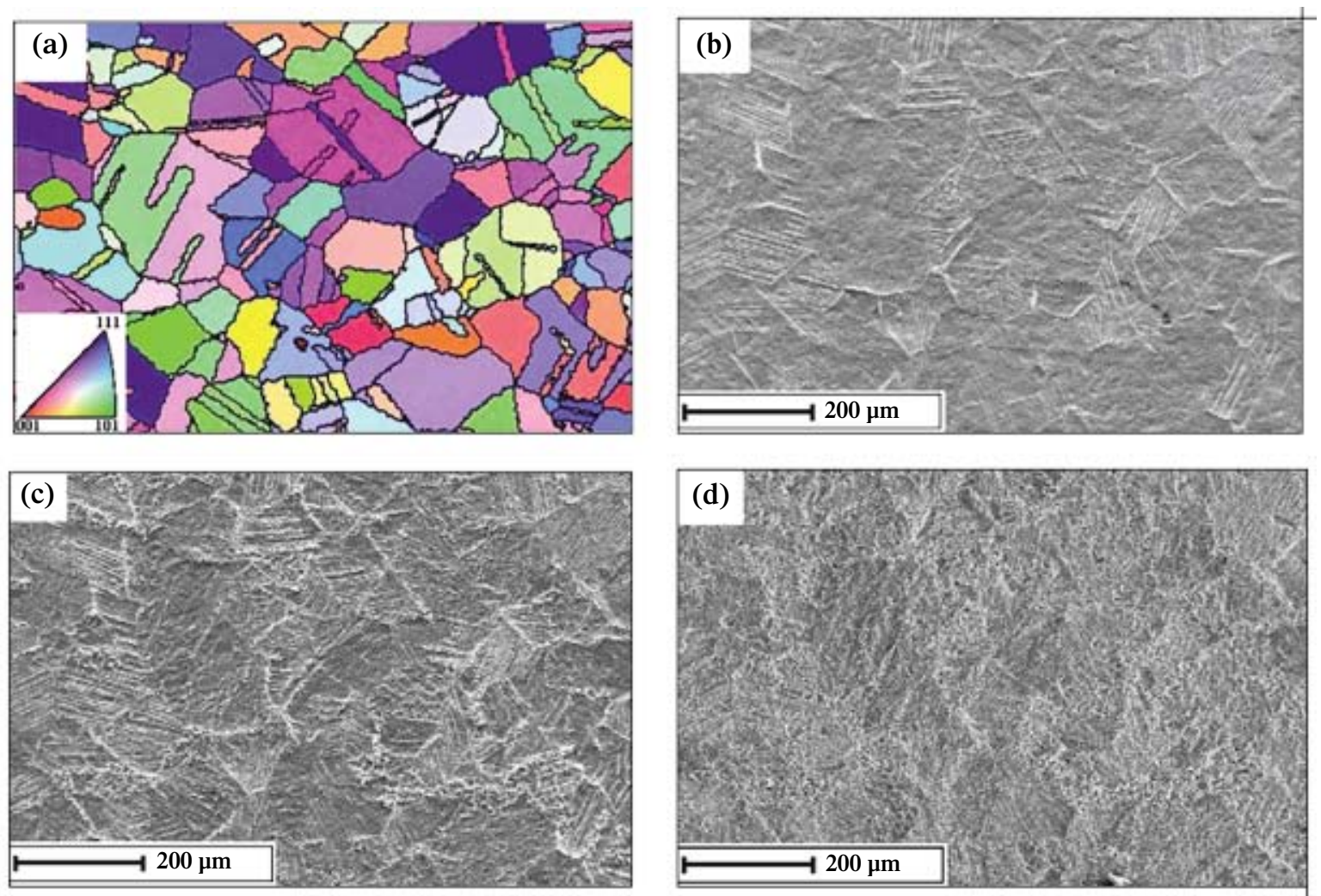

Figura 5. Evolución del proceso de desgaste por cavitación en el acero UNS S30403. (a) mapa EBSD correspondiente al área analizada y (b) a (d) micrografías SEM para 30, 180 y 360 minutos de ensayo respectivamente.

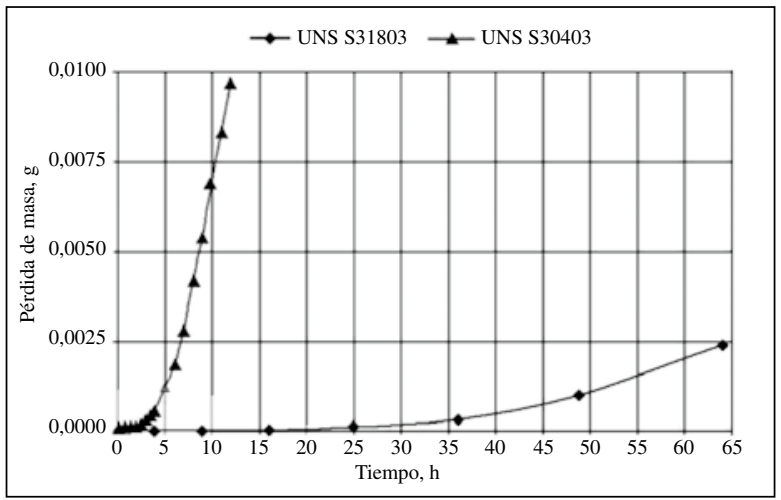

Figura 6. Curvas de pérdida de masa para los aceros UNS S31803 y UNS S30403.

Para el caso del acero UNS S30403 usado como material de comparación, es posible observar que el inicio del daño se presenta de forma similar al del acero UNS S31803 nitrurado, es decir, primero hay evidencia de deformación plástica en algunos granos, en los que se evidencia presencia de bandas de deslizamiento y en los límites de granos, especialmente en los de macla, los cuales son primeramente damnificados. Sólo que para este acero la falla por fractura frágil, que es el principal mecanismo de desgaste durante la mayor parte del tiempo en que duran los ensayos, es bastante severa para este material, haciendo que la superficie alcance un estado de deterioro similar al del acero UNS S31803 en apenas 6 horas de ensayo, tiempo para el cual el acero con nitrógeno no ha comenzado a presentar pérdida de masa medible, como se observa de las curvas de pérdida de masa de la Figura 6.

\section{CONCLUSIONES}

- La nitruración gaseosa en alta temperatura permitió transformar el acero UNS S31803, de fase dúplex, en un acero de una única fase austenítica. Donde la adición de nitrógeno en solución sólida no sólo permitió la eliminación de una estructura dúplex del material de partida, sino que favoreció la obtención de una estructura de granos con alto grado de textura, según la familia de planos (101) paralela a la superficie.

- Observando la evolución del daño por cavitación, grano a grano en el acero UNS S31803, se pudo constatar que el deterioro fue heterogéneo y que aquellos granos con planos de la familia (101), orientados aproximadamente paralelos a la superficie del material, son los que presentan mejor desempeño frente a este tipo de desgaste. 
- El inicio del daño, en los dos materiales ensayados, se presentó en la forma de deformación plástica (presencia de bandas de deslizamiento y cambios de rugosidad) y deterioro de los límites de grano, siendo la deformación plástica el principal mecanismo de daño para bajos tiempos de ensayo. Para tiempos de ensayo intermedios se tuvo una combinación de mecanismos de desgaste que consistieron en deformación plástica y fatiga de bajos ciclos seguida de fractura frágil. Ya para los mayores tiempos de ensayo el mecanismo principal de desgaste fue la fractura frágil.

De las curvas de pérdida de masa por cavitación en función del tiempo, fue posible determinar que el inicio del daño en el acero UNS S31803 es aproximadamente 10 veces más tardío que para el acero convencional, usado como material de comparación, es decir, el acero UNS S30403. Adicionalmente, el acero aleado con nitrógeno presentó también una resistencia al crecimiento del daño por EC muy superior a la exhibida por el material tradicional.

\section{AGRADECIMIENTOS}

A la Universidad Tecnológica de Pereira-Colombia y al grupo de investigación en Materiales de Ingeniería (GIMIUTP) por el apoyo para realizar estudios de doctorado en la Universidad de São Paulo (USP), Brasil.

Al grupo de investigación en Materiales para aplicaciones avanzadas de la USP, Brasil.

A la CAPES (Coordenação de Aperfeiçoamento de Pessoal de Nível Superior)-Brasília, Brasil. A COLCIENCIASInstituto Colombiano para el Desarrollo de la Ciencia y la Tecnología "Francisco José de Caldas", Colombia y a LASPAU por el apoyo económico para adelantar estudios de Doctorado en la USP, Brasil.

\section{REFERENCIAS}

[1] A.J. Schwartz, M. Kumar, D.P. Field and B.L. Adams. "Electron Backscatter Diffraction in Materials Science". Ed. Springer, $1^{\text {th }}$ Edition. New York, Estados Unidos. ISBN 030646487X. 2000.

[2] V. Randle. "Microtexture determination and its applications". Institute of Materials Minerals and Mining. $2^{\text {nd }}$ Edition. London, England. ISBN 9781902653839. 2003.

[3] D.J. Dingley and V. Randle. "Microtexture determination by electron back-scatter diffraction". Journal of Materials Science. Vol. 27, Issue 17, pp. 4545-4566. ISSN: 0022-2461. September, 1992.

[4] A.F. Padilha. "Utilização da técnica EBSD na caracterização microestrutural dos materiais".
Boletín Técnico da EPUSP, PMT, p. 16. São Paulo, Brasil. ISSN 1413-2176 .1999.

[5] R.P. Goehner and J.R. Michael. "Phase identification in EBSD using back-scattered electron Kikuchi patterns". J. Res. Natl. Inst. Stand. Tecnol. Vol. 101, Issue 3. ISSN 1044-677X. May/Juny, 1996.

[6] D.H. Mesa, C.M. Garzón e A.P. Tschiptschin. "Meso-anisotropia da deformação plástica e do desgaste durante a erosão por cavitação de um aço austenítico com alto teor de nitrogênio". $63^{\circ}$ Congresso anual da ABM. Santos, São Paulo, Brasil. 2008.

[7] D.H. Mesa, C.M. Garzón and A.P. Tschiptschin. "Mesoscale plasticity anisotropy at the earliest stages of cavitation-erosion damage of a high nitrogen austenitic stainless steel". Wear. Vol. 267, Issues 1-4, pp. 99-103. Juny, 2009.

[8] D.H. Mesa, C.M. Garzón y A.P. Tschiptschin. "Estudio del desgaste erosivo por cavitación a nivel del tipo de límites de grano y orientación cristalina de los granos en un acero inoxidable austenítico de alto nitrógeno". Scientia et Technica. Vol. 41, Issue 1, pp. 291-296. ISSN 0122-1701. Abril 2009.

[9] D.H. Mesa. "Principios de Tribología con énfasis en desgaste”. Ed. Universidad Tecnológica de Pereira, p. 305. Colombia. 2007.

[10] C.M. Garzón, J.F. Dos Santos and A.P Tschiptschin. "Improvement of the cavitation erosion resistance of an AISI 304L austenitic stainless steel by high temperature gas nitriding". Materials Science and Engineering A. Vol. 382, Issues 1-2, pp. 378-386. September, 2004.

[11] D.H. Mesa, A. Toro and A.P. Tschitschin. "The effect of testing temperature on corrosion-erosion resistance of martensitic stainless steels". Wear. Vol. 255, Issues 1-6, pp.139-145. September, 2003.

[12] C.M. Garzón, J.F Dos Santos e A.P Tschiptschin. "Melhora por meio de adição de nitrogênio da resistência a erosão por cavitação de aços inoxidáveis". 59 $9^{\circ}$ Congresso da ABM. São Paulo, Brasil. 2004.

[13] A.P. Tschiptschin and A. Toro. "Surface properties of high nitrogen steels". Conference HNS 2003, High Nitrogen Steel. Berna, Suiza. 2003.

[14] V.G. Gavriljuk and H. Berns. "High Nitrogen Steels". Springer-Verlag, $3^{\text {th }}$ Ed., p. 378. ISBN 3540664114. Alemania. 1999.

[15] H. Berns and S. Siebert. "High Nitrogen Austenitic Cases In Stainless Steels". ISIJ Int. Vol. 36, Issue 7, pp. 927-931. ISSN 1044-677X. Marzo, 1996.

[16] A. Krella. "Influence of cavitation intensity on X6CrNiTi18-10 stainless steel”. Wear. Vol. 258, Issues 11-12, pp. 1723-1731. ISSN 0043-1648. Juny, 2005. 